\title{
Autoimmune Hemolysis Anemia After Dihydroartemisinin and Piperaquine for Uncomplicated Plasmodium Falciparum Malaria
}

marion louvois

Centre Hospitalier Universitaire de Nice https://orcid.org/0000-0001-9198-2123

Loic Simon

CHU Nice: Centre Hospitalier Universitaire de Nice

Christelle Pomares

CHU Nice: Centre Hospitalier Universitaire de Nice

P-Y. Jeandel

CHU Nice: Centre Hospitalier Universitaire de Nice

\section{Elisa Demonchy}

CHU Nice: Centre Hospitalier Universitaire de Nice

Michel Carles

CHU Nice: Centre Hospitalier Universitaire de Nice

\section{Pascal Delaunay}

CHU Nice: Centre Hospitalier Universitaire de Nice

Johan Courjon ( $\nabla$ courjon.j@chu-nice.fr )

CHU Nice: Centre Hospitalier Universitaire de Nice

\section{Case report}

Keywords: Hemolytic anemia, malaria, artemisinin-based combination, artemisinin derivatives, autoimmune, direct antiglobulin test

Posted Date: June 29th, 2021

DOI: https://doi.org/10.21203/rs.3.rs-651064/v1

License: (c) (i) This work is licensed under a Creative Commons Attribution 4.0 International License. Read Full License 


\section{Autoimmune hemolysis anemia after dihydroartemisinin and piperaquine for uncomplicated Plasmodium falciparum malaria}

M. Louvois ${ }^{1}$, L. Simon ${ }^{2-3}$, C. Pomares ${ }^{2-3}$, P-Y. Jeandel ${ }^{4}$, E Demonchy, M Carles, P. Delaunay ${ }^{-3}$, J. Courjon ${ }^{3-5}$

1. Rhumatologie, Hôpital Pasteur 2, Centre Hospitalier Universitaire de Nice, Nice, France

2. Laboratoire de Parasitologie-Mycologie, Hôpital de l'Archet, Centre Hospitalier Universitaire de Nice, Nice, France

3. Université Côte d’Azur, Inserm 1065, C3M, Nice, France

4. Médecine Interne, Hôpital de l'Archet, Centre Hospitalier Universitaire de Nice, Nice, France

5. Infectiologie, Hôpital de l'Archet, Centre Hospitalier Universitaire de Nice, Nice, France

Corresponding author: COURJON Johan

address : 151 route de saint antoine de ginestière, Hôpital Archet 06200 Nice, France

email : courjon.j@chu-nice.fr

phone number : $+33(0) 492036003$

No Funding sources

No conflict of interest for all authors

All authors had access to the data and a role in writing the manuscript

Article type : case report 


\begin{abstract}
Background - Malaria is still an endemic disease in Africa with many imported cases in Europe. The standard treatment is intravenous artesunate for severe malaria and oral artemisinin-based combination therapy (ACT) for uncomplicated malaria. Delayed hemolytic anemia (DHA) after intravenous artesunate has been extensively described and guidelines recommend a biological monitoring until one month after the end of the treatment. The link with an autoimmune process is unsure. Nevertheless, cases with positive direct antiglobulin test (DAT) have been reported.

Conversely, DHA is not recognized as an adverse effect of oral ACT. Previously, only two cases of DHA occurring after oral ACT without intravenous artesunate administration have been reported.
\end{abstract}

Case presentation - We report the case of a 42 year old man returning from Togo. He was treated with dihydroartemisinin / piperaquine combination for an uncomplicated Plasmodium falciparum malaria. Nine days after the end of the treatment he developed hemolytic anemia with positive DAT. Eventually, the patient recovered after corticotherapy.

Conclusion - This is the first case report of autoimmune hemolytic anemia after treatment with dihydroartemisinin and piperaquine.

\title{
Keywords
}

Hemolytic anemia, malaria, artemisinin-based combination, artemisinin derivatives, autoimmune, direct antiglobulin test 


\section{Background}

Malaria is a mosquito-borne infectious disease endemic in tropical and subtropical areas. It is estimated that every year $12000-15000$ cases of malaria are imported into European Union countries (1). Among malaria parasites which can affect human, Plasmodium falciparum is the most frequent and is responsible for severe malaria and deaths. The recommended treatment of severe malaria is intravenous artesunate whereas, for the uncomplicated $P$. falciparum malaria, the treatment is oral artemisinin-based combination therapy (ACT) (2).

Risk of delayed hemolytic anemia (DHA) after intravenous artesunate is well acknowledged (3). It frequently occurs between day 7 and day 21 after the initiation of treatment. However, the pathophysiology of this adverse event is not entirely deciphered. The erythrocyte pitting process seems to be the main underlying factor (3). Some dead trophozoites are turned into pyknotic forms within the erythrocytes (4). These latest being damaged have a shorter lifetime (721 days) and are removed by the spleen. More recently, another mechanism of the anemia has been described, corresponding to drug induced immune hemolytic anemia (4). This is supported by detection of positive direct antiglobulin test (DAT) after artesunate treatment during DHA management and by the effectiveness of corticotherapy $(4,5,6)$. Guidelines recommend hemoglobin monitoring until one month after the end of the treatment but to date, DAT is not required. 
Conversely, DHA is not currently recognized as an adverse effect of oral ACT. To our knowledge, only two cases of DHA occurring after oral ACT without anterior intravenous artesunate administration, have been reported $(7,8)$. More recently Kurth et al. have described in a prospective study that hemolysis after oral ACT is more frequent than previously suspected, without hemolysis mechanism demonstration (pitting or auto immune process) (9).

Herein we present the case of an autoimmune hemolytic anemia after dihydroartemisinin and piperaquine treatment for uncomplicated $P$. falciparum malaria.

\section{$\underline{\text { Case presentation }}$}

December 31, 2019, a 42-year-old male patient visited the emergency department of Nice university hospital for abdominal pain.

His medical history was marked by a cured colon cancer ten years ago and by an uncomplicated $P$. falciparum malaria, diagnosed on 13 December 2019, ten days after a travel to Togo. He was treated by 3 days of dihydroartemisinin / piperaquine. The treatment was completed 15 days ago his visit to the emergency department.

At the emergency department, clinical examination showed a left hypochondrial pain and a cutaneous-mucosal pallor. 
The blood test showed a macrocytic anemia at $5.4 \mathrm{~g} / \mathrm{dl}$, with hemolysis criteria. Indeed, bilirubin was high ( $45 \mu \mathrm{mol} / 1)$, haptoglobin was under $0.10 \mathrm{~g} / \mathrm{l}, \mathrm{LDH}$ level was $2835 \mathrm{U} / \mathrm{L}$ and the reticulocytes were at $297 \mathrm{G} / \mathrm{L}$. The CT scan revealed a splenic infarction.

The patient was then hospitalized in the infectious diseases unit to investigate the cause of the hemolytic anemia.

Malaria parasitemia control was negative. The DAT was positive for the anti-C3d leading to the diagnosis of cold autoimmune hemolytic anemia.

A workup for autoimmune hemolytic anemia causes was made. Autoimmune assays (Antinuclear Antibodies (ANA), Extractable nuclear Antigen (ENA), rheumatoid factor) were negative. Blood protein electrophoresis and immunofixation were normal. EBV, hepatitis $\mathrm{B}, \mathrm{C}$ and E, HIV, HTLV1, Chlamydia pneumoniae and Coxiella burnetti serological tests were negative. CMV IgM and IgG antibodies were both positive but viral DNA detection in the blood by PCR was negative. Mycoplasma pneumoniae serology showed IgM and IgG antibodies positivity. Nevertheless, the serological monitoring didn't show seroconversion and therefore didn't support an acute infection. Finally, Parvovirus B19 IgM and IgG antibodies were close to the positive cut off but the patient didn't have any consistent symptoms (no flu like symptoms and no lymphocytosis). At the end, Mycoplasma pneumonia, Parvovirus B19 and CMV serologies were considered as non-specific reaction. 
We finally concluded to an autoimmune hemolytic anemia (AIHA) post dihydroartemisinin / piperaquine, complicated by splenic infarction.

At first, a red blood cells transfusion was performed before the diagnosis of AIHA was made and failed to improve the hemoglobin level, which stayed around $6 \mathrm{~g} / \mathrm{dl}$. Then an intravenous corticosteroid therapy (methylprednisolone) at $1 \mathrm{mg} / \mathrm{kg}$ was started and the hemoglobin increased to $8.3 \mathrm{~g} / \mathrm{dl}$ in 3 days and the hemolysis markers improved.

The patient was discharged with an oral corticosteroid therapy for 3 weeks. On the follow up red blood cells count 2 weeks after he was discharged, hemoglobin was $11.4 \mathrm{~g} / \mathrm{dl}$, haptoglobin 0,22, LDH $786 \mathrm{U} / \mathrm{L}$ and bilirubin $9 \mathrm{umol} / \mathrm{L}$.

\section{$\underline{\text { Discussion and conclusion }}$}

Autoimmune hemolytic anemia is an acquired hemolysis due to host's immune system acting against its own red cells antigens. Hemolysis is suggested by normo/macrocytic anemia, elevation of reticulocytes, of LDH and of unconjugated bilirubin whereas haptoglobin value is low. Then AIHA is confirmed by a positive DAT without any alternative causes. When DAT is positive for $\operatorname{IgG}$ or $\operatorname{IgG}+\mathrm{C} 3 \mathrm{~d}$, a diagnosis of warm AIHA can be made. A cold agglutinin syndrome would revealed positive anti-C3d only. Among AHAI, approximately $65 \%$ of patients have a warm AHAI, which is mainly caused by autoimmune disease and hematologic malignancy, while cold AHAI is mainly 
caused by infectious disease and hematologic malignancy (10). Drug dependent hemolytic anemia is another potential cause of AIHA.

The risk of delayed hemolytic anemia (DHA) after intravenous artesunate treatment is well acknowledged and more recently, artesunate-induced immune hemolytic anemia is also a described mechanism (4). Most of the drug-induced autoimmune hemolytic anemia are warm AHAI, which is not the case for our patient (11). Nevertheless, a retrospective study of post artesunate delayed hemolysis described four cases of AHAI with DAT positive on C3d only (4) and three other similar cases can be found in the literature $(6,12,13)$.

Conversely, DHA secondary to oral ACT has been less reported in literature. A case report has described DHA after treatment by arthemeterlumefantrine (AL) only and another case has been described after treatment by doxycycline and quinine first, then by $\operatorname{AL}(7,8)$. Both were P. falciparum malaria. In Kurth et al. prospective study in which 20 patients were included, eight patients treated by oral ACT presented posttreatment hemolysis, but it mainly occurs at a subclinical degree and no patient with anemia had a positive DAT(9).

Two mechanisms of DHA after treatment by artesunate are described: the pitting process, which is the most frequent; and more rarely, the drug-induced autoimmune hemolytic anemia. This second hypothesis has been suggested by increasingly cases with positive DAT and corticosteroids effectiveness (4). Nevertheless, systematic investigations have not been performed in most published cases and prospective clinical studies are needed. 
Among cases of DHA after oral ACT, one had a positive DAT and recovered with corticotherapy and the second had a negative DAT and recovered without corticotherapy $(7,8)$. Those cases of DHA occurred respectively after AL only and after doxycycline and quinine first, followed by AL.

Kurth et al. prospective study failed to demonstrate immune mediated hemolysis or a pitting process (9).

To our knowledge, our case report is the first about DHA in a patient treated exclusively with dihydroartemisinin and piperaquine and linked to an autoimmune process. A diagnosis of DHA after dihydroartemisinin / piperaquine was made after excluding differential diagnosis. The positive DAT for our patient linked the DHA to an autoimmune process, which was confirmed by the efficacy of the corticosteroid therapy.

In conclusion, our case supports the risk of DHA induced by oral artemisinin-based combination therapy. Only few cases of DHA after an oral artemisinin-based combination have been previously reported (two cases report and one prospective cohort) $(7,8,9)$. This prospective cohort demonstrates an underestimated risk of posttreatment hemolysis after oral ACT. Nevertheless, this hemolysis appears to be mild without autoimmune background.

Our case supports the drug-induced autoimmune hemolytic anemia rather than the pitting process to explain hemolysis.

Hemolysis monitoring after dihydroartemisinin / piperaquine should be reassessed. In case of DHA an autoimmune process can be involved. 


\section{$\underline{\text { Declarations }}$}

- Ethics approval and consent to participate: Not applicable

- Consent for publication: Written informed consent was obtained from the patient for the publication of this article.

- Availability of data and materials: Data sharing is not applicable to this article as no datasets were generated or analysed during the current study.

- Competing interests: The authors declare that they have no competing interests" in this section.

- Funding: Not applicable

- Authors' contributions: JC and ED have made the diagnosis and interpretated the data.

ML have made the acquisition of data.

JC and ML have drafted the work.

LS, CP, PJ, MC, PD substantively revised the work and helped to make the diagnosis.

All authors have approved the submitted version (and any substantially modified version that involves the author's contribution to the study

All authors have agreed both to be personally accountable for the author's own contributions and to ensure that questions related to the accuracy or integrity of any part of the work, even ones in which the author was not personally involved, are appropriately investigated, resolved, and the resolution documented in the literature.

- Acknowledgements: Not applicable 


\section{$\underline{\text { References }}$}

(1) Odolini, Silvia, Philippe Gautret, et Philippe Parola. « EPIDEMIOLOGY OF IMPORTED MALARIA IN THE MEDITERRANEAN REGION ». Mediterranean Journal of Hematology and Infectious Diseases 4, no 1 (7 mai 2012): e2012031. https://doi.org/10.4084/mjhid.2012.031.

(2) Guidelines for the Treatment of Malaria. 3rd éd. WHO Guidelines Approved by the Guidelines Review Committee. Geneva: World Health Organization, 2015. http://www.ncbi.nlm.nih.gov/books/NBK294440/.

(3) Aldámiz-Echevarría Lois, Teresa, Ana López-Polín, Francesca F. Norman, Begoña MongeMaillo, Rogelio López-Vélez, et Jose A. Perez-Molina. « Delayed Haemolysis Secondary to Treatment of Severe Malaria with Intravenous Artesunate: Report on the Experience of a Referral Centre for Tropical Infections in Spain ». Travel Medicine and Infectious Disease 15 (janvier 2017): 52-56. https://doi.org/10.1016/j.tmaid.2016.10.013.

(4) Camprubí, Daniel, Arturo Pereira, Natalia Rodriguez-Valero, Alex Almuedo, Rosauro Varo, Climent Casals-Pascual, Quique Bassat, Denis Malvy, et Jose Muñoz. « Positive Direct Antiglobulin Test in Post-Artesunate Delayed Haemolysis: More than a Coincidence? "

(5) Lebrun, Delphine, Thierry Floch, Aurélie Brunet, Gautier Julien, Juliette Romaru, Yohan N'Guyen, Joël Cousson, Aurélien Giltat, Dominique Toubas, et Firouzé Bani-Sadr. " Severe Post-Artesunate Delayed Onset Anaemia Responding to Corticotherapy: A Case Report ». Journal of Travel Medicine 25, $\mathrm{n}^{\circ} 1$ (1 janvier 2018). https://doi.org/10.1093/jtm/tax091.

(6) Raffray, Loic, Marie-Catherine Receveur, Mathilde Beguet, Pierre Lauroua, Thierry Pistone, et Denis Malvy. "Severe Delayed Autoimmune Haemolytic Anaemia Following Artesunate Administration in Severe Malaria: A Case Report ». Malaria Journal 13, $\mathrm{n}^{\circ} 1$ (2014): 398. https://doi.org/10.1186/1475-2875-13-398.

(7) De Nardo, P., A. Oliva, M. L. Giancola, P. Ghirga, P. Mencarini, M. Bibas, E. Nicastri, A. Antinori, et A. Corpolongo. « Haemolytic Anaemia after Oral Artemether-Lumefantrine Treatment in a Patient Affected by Severe Imported Falciparum Malaria ». Infection 41, n 4 (août 2013): 863-65. https://doi.org/10.1007/s15010-013-0451-x.

(8) Corpolongo, Angela, Pasquale De Nardo, Piero Ghirga, Elisa Gentilotti, Rita Bellagamba, Chiara Tommasi, Maria Grazia Paglia, Emanuele Nicastri, et Pasquale Narciso. « Haemolytic Anaemia in an HIV-Infected Patient with Severe Falciparum Malaria after Treatment with Oral Artemether-Lumefantrine ». Malaria Journal 11, n 1 (décembre 2012): 91. https://doi.org/10.1186/1475-2875-11-91.

(9) Kurth F, Lingscheid T, Steiner F, Stegemann MS, Bélard S, Menner N, et al. Hemolysis after Oral Artemisinin Combination Therapy for Uncomplicated Plasmodium falciparum Malaria. Emerging Infectious Diseases. 2016 Aug;22(8):1381-6.

(10) Hill, Anita, et Quentin A. Hill. « Autoimmune Hemolytic Anemia ». Hematology 2018, n 1 (30 novembre 2018): 382-89. https://doi.org/10.1182/asheducation-2018.1.382.

(11) Garratty, George. "Immune Hemolytic Anemia Associated with Drug Therapy ». Blood Reviews 24, n 4-5 (juillet 2010): 143-50. https://doi.org/10.1016/j.blre.2010.06.004.

(12) Jauréguiberry, Stéphane, Papa A. Ndour, Camille Roussel, Flavie Ader, Innocent Safeukui, Marie Nguyen, Sylvestre Biligui, et al. « Postartesunate Delayed Hemolysis Is a Predictable Event Related to the Lifesaving Effect of Artemisinins ». Blood 124, $n^{\circ} 2$ (10 juillet 2014): 167-75. https://doi.org/10.1182/blood-2014-02-555953.

(13) Singh, Nidhi, Sarvinder Singh, Bhaskar Shahbabu, Santosh Singh, Ajai Tentu, Anshu Kumar, et Vani Singh. «Artesunate-Induced Severe Autoimmune Hemolytic Anemia in Complicated 
Malaria ». Indian Journal of Critical Care Medicine 22, n 10 (octobre 2018): 753-56.

https://doi.org/10.4103/ijccm.IJCCM 29818. 